\title{
Multiple Periodic Solutions of Generalized Gause-Type Predator-Prey Systems with Nonmonotonic Numerical Responses and Impulse
}

\author{
Zhenguo Luo, ${ }^{1,2}$ Liping Luo, ${ }^{1}$ and Yunhui Zeng ${ }^{1}$ \\ ${ }^{1}$ Department of Mathematics, Hengyang Normal University, Hengyang, Hunan 421008, China \\ ${ }^{2}$ Department of Mathematics, National University of Defense Technology, Changsha 410073, China \\ Correspondence should be addressed to Zhenguo Luo; robert186@163.com
}

Received 2 October 2013; Accepted 20 November 2013; Published 17 February 2014

Academic Editors: N. Shanmugalingam and T. Tran

Copyright (C) 2014 Zhenguo Luo et al. This is an open access article distributed under the Creative Commons Attribution License, which permits unrestricted use, distribution, and reproduction in any medium, provided the original work is properly cited.

We consider an impulsive periodic generalized Gause-type predator-prey model with nonmonotonic numerical responses. Using the continuation theorem of coincidence degree theory, we present an easily verifiable sufficient condition on the existence of multiple periodic solutions. As corollaries, some applications are listed. In particular, our results extend and improve some known criteria.

\section{Introduction}

One of the powerful and effective methods on the existence of periodic solutions to periodic systems is the continuation method, which gives easily verifiable sufficient conditions. See Gaines and Mawhin [1] for detailed description of this method. In [2], Chen studied the following periodic predatorprey system with a Holling type IV functional response:

$$
\begin{aligned}
x^{\prime}(t)=x(t) & {[r(t)-a(t) x(t-\tau(t))} \\
& \left.-\frac{b(t) x\left(t-\sigma_{1}(t)\right)}{\left(x^{2}(t) / m\right)+x(t)+n}\right], \\
y^{\prime}(t)=y(t)[-d(t) & c(t) x\left(t-\sigma_{2}(t)\right) \\
& \left.+\frac{c\left(t-\sigma_{2}(t)\right)+n}{\left.\left(x^{2}\left(t-\sigma_{2}(t)\right)\right) / m\right)+x(t)}\right]
\end{aligned}
$$

where $r, d, \tau, \sigma_{1}, \sigma_{2} \in C(R, R)$ and $a, b, c \in C\left(R, R^{+}\right)$are $\omega$-periodic functions with $\int_{0}^{\omega} r(t) \mathrm{d} t>0$ and $\int_{0}^{\omega} d(t) \mathrm{d} t>0$, and $m, n$ are positive constants. The results on the existence of multiple periodic solutions have been obtained by employing the continuation method. There are some works following this direction. See, for example, [3-6].

To generalize Chen's results, Ding and Jiang [4] considered the following periodic Gause-type predator-prey system with time delays:

$$
\begin{gathered}
x^{\prime}(t)=x(t) f(t, x(t-\tau(t))) \\
-g(t, x(t)) y\left(t-\sigma_{1}(t)\right), \\
y^{\prime}(t)=y(t)\left[-d(t)+h\left(t, x\left(t-\sigma_{2}(t)\right)\right)\right],
\end{gathered}
$$

where $\tau, \sigma_{1}, \sigma_{2}$, and $d$ are continuous $\omega$-periodic functions with $\int_{0}^{\omega} d(t) \mathrm{d} t>0$. They also afforded verifiable criteria for the existence of multiple positive periodic solutions for the system (2) when the numerical response function $h$ is nonmonotonic. Their results improve and supplement those in [2].

As we know, in population dynamics, many evolutionary processes experience short-time rapid change after undrgoing relatively long smooth variation. For example, the harvesting and stocking occur at fixed time, and some species usually immigrate at the same time every year. Incorporating these phenomena gives us impulsive differential equations. 
For theory of impulsive differential equations, we refer to [7$16]$.

Based on the previous ideas, in [17], Wang, Dai, and Chen considered the following impulse predator-prey system with a Holling type IV functional response:

$$
\begin{gathered}
x^{\prime}(t)=x(t)[r(t)-a(t) x(t-\tau(t)) \\
\left.-\frac{b(t) x\left(t-\sigma_{1}(t)\right)}{\left(x^{2}(t) / m\right)+x(t)+n}\right], \\
y^{\prime}(t)=y(t)\left[\begin{array}{l}
-d(t) \\
\left.+\frac{c(t) x\left(t-\sigma_{2}(t)\right)}{\left(\left(x^{2}\left(t-\sigma_{2}(t)\right)\right) / m\right)+x\left(t-\sigma_{2}(t)\right)+n}\right], \\
\Delta x(t)=x\left(t^{+}\right)-x(t)=c_{1 k} x(t), \\
\Delta y(t)=y\left(t^{+}\right)-y(t)=c_{2 k} y(t), \quad t=t_{k},
\end{array}\right.
\end{gathered}
$$

where the assumptions on $r, d, \tau, \sigma_{1}, \sigma_{2}, a, b, c, m, n$ are the same as $(1), c_{j k} \in(-1, \infty)(j=1,2, k \in N=\{1,2, \ldots\})$, $\left\{t_{k}\right\}_{k \in N}$ is a strictly increasing sequence with $t_{1}>0$, and $\lim _{k \rightarrow \infty} t_{k}=\infty$. Further, there exist a $q \in N$ such that $c_{j(k+q)}=c_{j k}(j=1,2)$ and $t_{k+q}=t_{k}+\omega$ for $k \in N$. By employing the continuation theorem, they presented sufficient conditions on the existence of two positive periodic solutions to system (3).

In this paper, we will consider the following Gause-type predator-prey systems with impulse and time delays:

$$
\begin{gathered}
x^{\prime}(t)=x(t) f(t, x(t-\tau(t)))-g(t, x(t)) y\left(t-\sigma_{1}(t)\right), \\
y^{\prime}(t)=y(t)\left[-d(t)+h\left(t, x\left(t-\sigma_{2}(t)\right)\right)\right], \quad t \neq t_{k}, \\
\Delta x(t)=x\left(t^{+}\right)-x(t)=c_{1 k} x(t), \\
\Delta y(t)=y\left(t^{+}\right)-y(t)=c_{2 k} y(t), \quad t=t_{k},
\end{gathered}
$$

where the assumptions on $d, \tau, \sigma_{1}, \sigma_{2}, c_{j k}$, and $t_{k}$ are the same as (3). $x$ and $y$ are the prey and the predator population size, respectively. The function $f$ is the growth rate of the prey in the absence of the predator and $d$ is the death rate of the predator. The function $g$, called functional response of predator to prey, describes the change in the rate of exploitation of prey by a predator as a result of a change in the prey density. The function $h$, called numerical response of predator to prey, describes the change in reproduction rate with change in the prey density.
In general, the response function is monotone (see [1820]). However, there is nonmonotonic response occurrence; see Kuang and Beretta [21]. The so-called Monod-Haldane function in (5)

$$
h(u)=\frac{c u}{\left(u^{2}+m u+n\right)},
$$

has been proposed and used to model; see [22]. Sokol and Howell [23] proposed a simplified Monod-Haldane function of the form in (6)

$$
f(u)=\frac{c u}{\left(m^{2}+u^{2}\right)} .
$$

Throughout this paper, we assume the following:

$\left(H_{1}\right) f(t, u), g(t, u)$, and $h(t, u)$ are continuous functions and $\omega$-periodic with respect to $t ; \partial f / \partial u, \partial g / \partial u$, and $\partial h / \partial u$ are also continuous functions;

$\left(H_{2}\right)$ there exists a positive constant $\alpha$ such that $\int_{0}^{\omega} f(t, u) \mathrm{d}$ $t>0$ for $u \in(0, \alpha)$; there also exists a continuous $\omega$-periodic function $r(t)$ such that $\int_{0}^{\omega} r(t) \mathrm{d} t>0$ and $f(t, u) \leq r(t)$ for $t \in R, u \geq 0$;

$\left(H_{3}\right) g(t, 0)=0, g(t, u)>0$ for $t \in R, u>0$; there exists a positive constant $\mu$ such that $g(t, u) \leq \mu u$ for $t \in R$, $u>0$

$\left(H_{4}\right) h(t, 0)=0, \lim _{u \rightarrow \infty} h(t, u)=0$; there exists a positive constant $p$ such that $(u-p)(\partial h / \partial u)<0$ for $t \in$ $R, u \neq p$;

$$
\begin{aligned}
& \left(H_{5}\right) \int_{0}^{\omega} d(t) \mathrm{d} t-\sum_{k=1}^{q} \ln \left(1+c_{2 k}\right)<\sup _{u \geq 0} \int_{0}^{\omega} h(t, u) \mathrm{d} t \\
& \left(H_{6}\right) \inf _{u \in(0, \alpha)} \int_{0}^{\omega} f(t, u) d t+\sum_{k=1}^{q} \ln \left(1+c_{1 k}\right)>0 .
\end{aligned}
$$

Remark 1. The assumption $\left(H_{4}\right)$ demonstrates that numerical response function $h$ is non-monotonic. The function in (5) and the function $f$ in (6) both satisfy the condition $\left(H_{4}\right)$.

The main purpose of the present paper is, by using the coincidence theory developed by Gaines and Mawhin [1], to establish the sufficient conditions for the existence of multiple positive periodic solutions of system (4) when $h$ is a nonmonotonic numerical response function. As corollaries, some applications are listed. In particular, our results extend and improve some known criteria.

\section{Preliminaries}

The method to be used in this paper involves the applications of the continuation theorem of coincidence degree. For the readers' convenience, we introduce a few concepts and results about the coincidence degree as follows.

Let $X, Z$ be two real Banach spaces, let $L: \operatorname{Dom} L \subset X \rightarrow$ $Z$ be a linear mapping, and let $N: X \rightarrow Z$ be a continuous mapping. The mapping $L$ will be called a Fredholm mapping of index zero if $\operatorname{dim} \operatorname{Ker} L=$ condim $\operatorname{Im} L<+\infty$ and $\operatorname{Im} L$ is 
closed in $Z$. If $L$ is a Fredholm mapping of index zero and there exist continuous projectors $P: X \rightarrow X$ and $Q: Z \rightarrow$ $Z$ such that $\operatorname{Im} P=\operatorname{Ker} L, \operatorname{Ker} Q=\operatorname{Im} L=\operatorname{Im}(I-Q)$, it follows that $\left.L\right|_{\text {Dom } L \cap K e r P}:(I-P) X \rightarrow \operatorname{Im} L$ is invertible; we denote the inverse of that map by $K_{p}$. If $\Omega$ is an open bounded subset of $X$, the mapping $N$ will be called L-compact on $\bar{\Omega}$ if $Q N(\bar{\Omega})$ is bounded and $K_{p}(I-Q) N: \bar{\Omega} \rightarrow X$ is compact. Since $\operatorname{Im} Q$ is isomorphic to $\operatorname{Ker} L$, there exist isomorphisms $J: \operatorname{Im} Q \rightarrow \operatorname{Ker} L$. Let $P C_{\omega}$ denote the space of $\omega$-periodic periodic functions $\Psi: J \rightarrow R$ which are continuous for $t \neq t_{k}$, are continuous from the left for $t \in R$, and have discontinuities of the first kind at point $t=t_{k}$. We also denote $P C_{\omega}^{1}=\left\{\Psi \in P C_{\omega}: \Psi^{\prime} \in P C_{\omega}\right\}$.

Lemma 2 (see continuation theorem [1]). Let $X$ and $Z$ be two Banach spaces and let $L: \operatorname{Dom} L \subset X \rightarrow Z$ be a Fredholm operator with index zero. $\Omega \subset X$ is an open bounded set, and let $N: \bar{\Omega} \rightarrow Z$ be L-compact on $\bar{\Omega}$. Suppose that

(a) $x$ of $L x=\lambda N x$ for such $\lambda \in(0,1)$, and $x \notin$ $\partial \Omega \bigcap \operatorname{Dom} L$

(b) $Q N x \neq 0$ for each $x \in \partial \Omega \cap \operatorname{ker} L$;

(c) $\operatorname{deg}\{J Q N, \Omega \cap \operatorname{Ker} L, 0\} \neq 0$.

Then, the equation $L x=N x$ has at least one solution lying in $\operatorname{Dom} L \cap \bar{\Omega}$.

Lemma 3 (see $[12,17])$. Suppose that $\psi \in P C_{\omega}^{1},[0, \omega] \cap\left\{t_{k}\right\}=$ $t_{1}, t_{2}, \ldots, t_{q}$. Then

$$
\begin{aligned}
\sup _{s \in[0, \omega]} \psi(s)-\inf _{s \in[0, \omega]} \psi(s) \\
\leq \frac{1}{2}\left[\int_{0}^{\omega}|\dot{\psi}(s)| d s+\sum_{k=1}^{q}\left|\Delta \psi\left(t_{k}\right)\right|\right] .
\end{aligned}
$$

For convenience, we make change of variables $x(t)=$ $\exp \left\{x_{1}(t)\right\}, y(t)=\exp \left\{x_{2}(t)\right\}$, and the system (4) is reformulated as

$$
\begin{gathered}
x_{1}^{\prime}(t)=f\left(t, e^{x_{1}(t-\tau(t))}\right)-g\left(t, e^{x_{1}(t)}\right) e^{x_{2}\left(t-\sigma_{1}(t)\right)-x_{1}(t)}, \\
x_{2}^{\prime}(t)=h\left(t, e^{x_{1}\left(t-\sigma_{2}(t)\right)}\right)-d(t), \quad t \neq t_{k}, \\
\Delta x_{1}(t)=\ln \left(1+c_{1 k}\right), \\
\Delta x_{2}(t)=\ln \left(1+c_{2 k}\right), \quad t=t_{k} .
\end{gathered}
$$

It is easy to see that if system (8) has one $\omega$-periodic solution $\left(x_{1}^{*}(t), x_{2}^{*}(t)\right)^{T}$, then $\left(x^{*}(t), y^{*}(t)\right)^{T}=\left(\exp \left\{x_{1}^{*}(t)\right\}\right.$, $\left.\exp \left\{x_{2}^{*}(t)\right\}\right)^{T}$ is a positive $\omega$-periodic solution of system (4). Therefore, to complete the proof, it suffices to show that system (8) has multiple $\omega$-periodic solutions.

We take

$$
\begin{gathered}
X=\left\{x=\left(x_{1}, x_{2}\right)^{T} \mid x_{i} \in P C_{\omega}, i=1,2\right\}, \\
Z=X \times R^{2 q},
\end{gathered}
$$

and define

$$
\begin{gathered}
\|x\|_{X}=\sum_{i=1}^{2} \sup _{t \in[0, \omega]}\left|x_{i}(t)\right|, \quad x=\left(x_{1}, x_{2}\right)^{T} \in X, \\
\|z\|_{Z}=\|x\|_{X}+\|y\|, \quad z=(x, y) \in Z,
\end{gathered}
$$

where $\|\cdot\|$ is the Euclidean norm of $R^{2 q}$. Then $X$ and $Z$ are Banach spaces.

Let

$$
\operatorname{Dom} L=\left\{x=\left(x_{1}, x_{2}\right)^{T} \mid x_{i} \in P C_{\omega}^{1}, i=1,2\right\},
$$

$L: \operatorname{Dom} L \subset X \longrightarrow Z, \quad L x=\left(x^{\prime}, \Delta x\left(t_{1}\right), \ldots, \Delta x\left(t_{q}\right)\right)$,

and $N: X \rightarrow Z$ with

$$
N x=\left(\left[\begin{array}{l}
f\left(t, e^{x_{1}(t-\tau(t))}\right)-g\left(t, e^{x_{1}(t)}\right) e^{x_{2}\left(t-\sigma_{1}(t)\right)-x_{1}(t)} \\
h\left(t, e^{x_{1}\left(t-\sigma_{2}(t)\right)}\right)-d(t)
\end{array}\right],\left\{\left(\begin{array}{l}
\ln \left(1+c_{1 k}\right) \\
\ln \left(1+c_{2 k}\right)
\end{array}\right)\right\}_{k=1}^{q}\right) .
$$

It is not difficult to show that

$$
\operatorname{Ker} L=\left\{x \in X \mid x=\varphi \in R^{2}\right\}
$$

$$
\operatorname{Im} L=\left\{z=\left(\varphi, c_{1}, \ldots, c_{q}\right) \in Z \mid \int_{0}^{\omega} \varphi(s) d s+\sum_{k=1}^{q} c_{k}=0\right\}
$$

and $\operatorname{dim} \operatorname{Ker} L=2=\operatorname{codim} \operatorname{Im} L$. So, $\operatorname{Im} L$ is closed in $Z$, and $L$ is a Fredholm mapping of index zero. Take

$$
P x=\frac{1}{\omega} \int_{0}^{\omega} x(t) d t, \quad x \in X
$$

$$
\begin{aligned}
Q z & =Q\left(\varphi, c_{1}, \ldots, c_{q}\right) \\
& =\left(\frac{1}{\omega}\left[\int_{0}^{\omega} \varphi(s) d s+\sum_{k=1}^{q} c_{k}\right],(0,0, \ldots, 0)_{2 \times q}\right) .
\end{aligned}
$$


It is trivial to show that $P, Q$ are continuous projectors such that

$$
\operatorname{Im} P=\operatorname{Ker} L, \quad \operatorname{Ker} Q=\operatorname{Im} L=\operatorname{Im}(I-Q),
$$

and, hence, the generalized inverse $K_{P}$ exists. In the following part, we first devote ourselves to deriving the explicit expression of $K_{P}: \operatorname{Im} L \rightarrow \operatorname{Ker} P \cap \operatorname{Dom} L$. Taking $z=$ $\left(\varphi, c_{1}, \ldots, c_{q}\right) \in \operatorname{Im} L$, then exists an $x \in \operatorname{Dom} L \subset X$ such that

$$
\begin{gathered}
x^{\prime}(t)=\varphi(t), \quad t \neq t_{k}, \\
\Delta x(t)=c_{k}, \quad t=t_{k}, \quad k=1,2, \ldots, q .
\end{gathered}
$$

Then direct integration produces

$$
x(t)=\int_{0}^{t} \varphi(s) d s+\sum_{t>t_{k}} c_{k}+x(0) .
$$

Note that $x(t) \in \operatorname{Ker} P$; that is, $\int_{0}^{\omega} x(s) d s=0$, which, together with (17), implies

$$
\int_{0}^{\omega} \int_{0}^{t} \varphi(s) d s d t+\int_{0}^{\omega} \sum_{t>t_{k}} c_{k} d t+\omega x(0)=0
$$

then

$$
\begin{aligned}
x(t)= & \int_{0}^{t} \varphi(s) d s+\sum_{t>t_{k}} c_{k}-\frac{1}{\omega} \int_{0}^{\omega} \int_{0}^{t} \varphi(s) d s d t \\
& -\sum_{k=1}^{q} c_{k}+\frac{1}{\omega} \sum_{k=1}^{q} c_{k} t_{k},
\end{aligned}
$$

that is,

$$
\begin{aligned}
K_{P} z= & \int_{0}^{t} \varphi(s) d s+\sum_{t>t_{k}} c_{k}-\frac{1}{\omega} \int_{0}^{\omega} \int_{0}^{t} \varphi(s) d s d t \\
& -\sum_{k=1}^{q} c_{k}+\frac{1}{\omega} \sum_{k=1}^{q} c_{k} t_{k} .
\end{aligned}
$$

Thus, for $x \in X$,

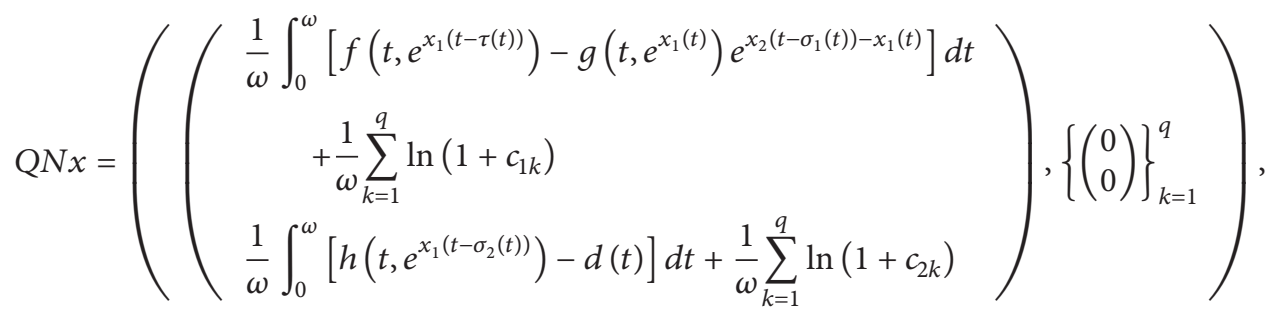

$$
\begin{aligned}
& K_{P}(I-Q) N x=\left(\begin{array}{c}
\int_{0}^{t}\left[f\left(u, e^{x_{1}(u-\tau(u))}\right)-g\left(u, e^{x_{1}(u)}\right) e^{x_{2}\left(u-\sigma_{1}(u)\right)-x_{1}(u)}\right] d u \\
+\sum_{t>t_{k}} \ln \left(1+c_{1 k}\right) \\
\int_{0}^{t}\left[h\left(u, e^{x_{1}\left(u-\sigma_{2}(u)\right)}\right)-d(u)\right] d u+\sum_{t>t_{k}} \ln \left(1+c_{2 k}\right)
\end{array}\right) \\
& -\left(\begin{array}{c}
\frac{1}{\omega} \int_{0}^{\omega} \int_{0}^{t}\left[f\left(u, e^{x_{1}(u-\tau(u))}\right)-g\left(u, e^{x_{1}(u)}\right) e^{x_{2}\left(u-\sigma_{1}(u)\right)-x_{1}(u)}\right] d u d t \sum_{k=1}^{q} \ln \left(1+c_{1 k}\right) \\
-\frac{1}{\omega} \sum_{k=1}^{q} \ln \left(1+c_{1 k}\right) t_{k} \\
\frac{1}{\omega} \int_{0}^{\omega} \int_{0}^{t}\left[h\left(u, e^{x_{1}\left(u-\sigma_{2}(u)\right)}\right)-d(u)\right] d u d t+\sum_{k=1}^{q} \ln \left(1+c_{2 k}\right)-\frac{1}{\omega} \sum_{k=1}^{q} \ln \left(1+c_{2 k}\right) t_{k}
\end{array}\right) \\
& -\left(\begin{array}{c}
\left(\frac{t}{\omega}-\frac{1}{2}\right) \int_{0}^{\omega}\left[f\left(u, e^{x_{1}(u-\tau(u))}\right)-g\left(u, e^{x_{1}(u)}\right) e^{x_{2}\left(u-\sigma_{1}(u)\right)-x_{1}(u)}\right] d u \\
+\left(\frac{t}{\omega}-\frac{1}{2}\right) \sum_{k=1}^{q} \ln \left(1+c_{1 k}\right) \\
\left(\frac{t}{\omega}-\frac{1}{2}\right) \int_{0}^{\omega}\left[h\left(u, e^{x_{1}\left(u-\sigma_{2}(u)\right)}\right)-d(u)\right] d u+\left(\frac{t}{\omega}-\frac{1}{2}\right) \sum_{k=1}^{q} \ln \left(1+c_{2 k}\right)
\end{array}\right) .
\end{aligned}
$$


Clearly, $Q N$ and $K_{P}(I-Q) N$ are continuous. By applying Ascoli-Arzela theorem, one can easily show that $Q N(\bar{\Omega})$, $K_{P}(I-Q) N(\bar{\Omega})$ are relatively compact for any open bounded set $\Omega \subset X$. Moreover, $Q N(\bar{\Omega})$ is obviously bounded. Thus, $N$ is $L$-compact on $\bar{\Omega}$ for any open bounded set $\Omega \subset X$.

In what follows, we shall use the notations

$$
\begin{gathered}
\bar{a}=\frac{1}{\omega} \int_{0}^{\omega} a(t) d t, \quad \overline{|a|}=\frac{1}{\omega} \int_{0}^{\omega}|a(t)| d t, \\
B(u)=\frac{1}{\omega} \int_{0}^{\omega} b(t, u) d t
\end{gathered}
$$

where $a(t)$ is a continuous $\omega$-periodic function, $b(t, u)$ is a continuous function, and $\omega$-periodic with respect to $t$.

We also set

$$
\begin{gathered}
D=\bar{d}+\overline{|d|}, \quad R=\bar{r}+\overline{|r|}, \quad c_{j}=\sum_{k=1}^{q} \ln \left(1+c_{j k}\right), \\
\rho_{1}=\sum_{k=1}^{q}\left[\ln \left(1+c_{1 k}\right)+\left|\ln \left(1+c_{1 k}\right)\right|\right] \\
\rho_{2}=\sum_{k=1}^{q}\left[-\ln \left(1+c_{2 k}\right)+\left|\ln \left(1+c_{2 k}\right)\right|\right] .
\end{gathered}
$$

\section{Existence of Multiple Positive Periodic Solutions}

By $\left(H_{2}\right)$ and $\left(H_{4}\right)$, we have

$$
\begin{gathered}
(u-p) H^{\prime}(u)=\frac{1}{\omega} \int_{0}^{\omega}(u-p) \frac{\partial h}{\partial u}(t, u)<0, \\
H(0)=0, \quad \lim _{u \rightarrow \infty} H(u)=0 .
\end{gathered}
$$

Then $H(u)$ is strictly increasing on $[0, p]$ and strictly decreasing on $[p,+\infty)$. By $\left(H_{4}\right)$ and $\left(H_{5}\right)$, if

$$
\left(H_{7}\right) \bar{d} \omega-c_{2}>0
$$

then, the equation

$$
H(u)=\bar{d}-\frac{c_{2}}{\omega}
$$

has two distinct positive solutions, namely, $u_{-}, u_{+}$. Without loss of generality, we suppose that $u_{-}<u_{+}$, and then $u_{-}<$ $p<u_{+}$.

Now, we are ready to state and prove our main result.

Theorem 4. In addition to the conditions $\left(H_{1}\right)-\left(H_{7}\right)$, suppose further that the following conditions hold:

$$
\begin{aligned}
& \left(H_{8}\right) u_{-} e^{R \omega+\rho_{1}}<u_{+} ; \\
& \left(H_{9}\right) u_{+} e^{(1 / 2)\left(R \omega+\rho_{1}\right)}<\alpha .
\end{aligned}
$$

Then, system (4) has at least two $\omega$-periodic solutions with strictly positive components.

Proof. By Lemma 2, we need to search for two appropriate open bounded subsets $\Omega_{i} \subset X(i=1,2)$. Considering the corresponding operator equation $L x=\lambda N x, \lambda \in(0,1)$, we have

$$
\begin{gathered}
x_{1}^{\prime}(t)=\lambda\left[f\left(t, e^{x_{1}(t-\tau(t))}\right)-g\left(t, e^{x_{1}(t)}\right) e^{x_{2}\left(t-\sigma_{1}(t)\right)-x_{1}(t)}\right], \\
x_{2}^{\prime}(t)=\lambda\left[h\left(t, e^{x_{1}\left(t-\sigma_{2}(t)\right)}\right)-d(t)\right], \quad t \neq t_{k}, \\
\Delta x_{1}(t)=\lambda \ln \left(1+c_{1 k}\right), \\
\Delta x_{2}(t)=\lambda \ln \left(1+c_{2 k}\right), \quad t=t_{k} .
\end{gathered}
$$

Let $x(t)=\left(x_{1}(t), x_{2}(t)\right)^{T} \in X$ be a $\omega$-periodic solution of system (26) for a certain $\lambda \in(0,1)$, integrating both sides of the first and second equations of (26) over the interval $[0, \omega]$; we obtain

$$
\begin{gathered}
\int_{0}^{\omega} f\left(t, e^{x_{1}(t-\tau(t))}\right)-g\left(t, e^{x_{1}(t)}\right) e^{x_{2}\left(t-\sigma_{1}(t)\right)-x_{1}(t)} d t=-c_{1}, \\
\int_{0}^{\omega} h\left(t, e^{x_{1}\left(t-\sigma_{2}(t)\right)}\right) d t=\bar{d} \omega-c_{2} .
\end{gathered}
$$

From the first equation of (27), we have

$$
\begin{aligned}
& \int_{0}^{\omega}\left[r(t)-f\left(t, e^{x_{1}(t-\tau(t))}\right)+g\left(t, e^{x_{1}(t)}\right) e^{x_{2}\left(t-\sigma_{1}(t)\right)-x_{1}(t)}\right] d t \\
& \quad=\bar{r} \omega+c_{1} .
\end{aligned}
$$

It follows from (26)-(28) that

$$
\begin{aligned}
& \int_{0}^{\omega}\left|x_{1}^{\prime}(t)\right| d t \leq R \omega+c_{1}, \\
& \int_{0}^{\omega}\left|x_{2}^{\prime}(t)\right| d t \leq D \omega-c_{2} .
\end{aligned}
$$

Note that $x(t)=\left(x_{1}(t), x_{2}(t)\right) \in X$; then there exist $\xi_{i}, \eta_{i} \in$ $[0, \omega](i=1,2)$ such that

$$
x_{i}\left(\xi_{i}\right)=\inf _{t \in[0, \omega]} x_{i}(t), \quad x_{i}\left(\eta_{i}\right)=\sup _{t \in[0, \omega]} x_{i}(t), \quad i=1,2 .
$$

By (27), (30), and the monotonicity of $h$ and $H$, we will show that $x_{1}\left(\xi_{1}\right)$ and $x_{1}\left(\eta_{1}\right)$ can not simultaneously lie in $\left(-\infty, \ln u_{-}\right),\left(\ln u_{-}, \ln u_{+}\right)$, or $\left(\ln u_{+},+\infty\right)$. In fact, if $x_{1}\left(\xi_{1}\right) \leq$ $x_{1}\left(\eta_{1}\right)<\ln u_{-}$, then

$$
\begin{aligned}
\bar{d}-\frac{c_{2}}{\omega} & =\frac{1}{\omega} \int_{0}^{\omega} h\left(t, e^{x_{1}\left(t-\sigma_{2}(t)\right)}\right) d t \\
& \leq H\left(e^{x_{1}\left(\eta_{1}\right)}\right)<H\left(u_{-}\right)=\bar{d}-\frac{c_{2}}{\omega} .
\end{aligned}
$$


This is a contradiction. If $\ln u_{+}<x_{1}\left(\xi_{1}\right) \leq x_{1}\left(\eta_{1}\right)$, then

$$
\begin{aligned}
\bar{d}-\frac{c_{2}}{\omega} & =\frac{1}{\omega} \int_{0}^{\omega} h\left(t, e^{x_{1}\left(t-\sigma_{2}(t)\right)}\right) d t \\
& \leq H\left(e^{x_{1}\left(\xi_{1}\right)}\right)<H\left(u_{+}\right)=\bar{d}-\frac{c_{2}}{\omega} .
\end{aligned}
$$

This is a contradiction. If In $u_{-}<x_{1}\left(\xi_{1}\right) \leq x_{1}\left(\eta_{1}\right)<\ln u_{+}$, then

$$
\begin{aligned}
\bar{d}-\frac{c_{2}}{\omega} & =\frac{1}{\omega} \int_{0}^{\omega} h\left(t, e^{x_{1}\left(t-\sigma_{2}(t)\right)}\right) d t \\
& \geq \min \left\{H\left(e^{x_{1}\left(\xi_{1}\right)}\right), H\left(e^{x_{1}\left(\eta_{1}\right)}\right)\right\}>H\left(u_{ \pm}\right) \\
& =\bar{d}-\frac{c_{2}}{\omega} .
\end{aligned}
$$

This is also a contradiction. Consequently, the distributions of $x_{1}\left(\xi_{1}\right)$ and $x_{1}\left(\eta_{1}\right)$ only have the following two cases.

Case $1\left(x_{1}\left(\xi_{1}\right) \leq \ln u_{-} \leq x_{1}\left(\eta_{1}\right)\right)$. From the first equation of (29) and Lemma 3, we have

$$
\begin{aligned}
& x_{1}(t) \geq x_{1}\left(\eta_{1}\right)-\frac{1}{2}\left(R \omega+\rho_{1}\right) \geq \ln u_{-}-\frac{1}{2}\left(R \omega+\rho_{1}\right):=\alpha_{1}, \\
& x_{1}(t) \leq x_{1}\left(\xi_{1}\right)+\frac{1}{2}\left(R \omega+\rho_{1}\right) \leq \ln u_{-}+\frac{1}{2}\left(R \omega+\rho_{1}\right):=\alpha_{2} .
\end{aligned}
$$

Case $2\left(x_{1}\left(\xi_{1}\right) \leq \operatorname{In} u_{+} \leq x_{1}\left(\eta_{1}\right)\right)$. From the first equation of (29) and Lemma 3, we also have

$$
\begin{aligned}
& x_{1}(t) \geq x_{1}\left(\eta_{1}\right)-\frac{1}{2}\left(R \omega+\rho_{1}\right) \geq \ln u_{+}-\frac{1}{2}\left(R \omega+\rho_{1}\right):=\alpha_{3}, \\
& x_{1}(t) \leq x_{1}\left(\xi_{1}\right)+\frac{1}{2}\left(R \omega+\rho_{1}\right) \leq \ln u_{+}+\frac{1}{2}\left(R \omega+\rho_{1}\right):=\alpha_{4} .
\end{aligned}
$$

By $\left(H_{8}\right)$, we know

$$
\alpha_{1}<\operatorname{In} u_{-}<\alpha_{2}<\alpha_{3}<\ln u_{+}<\alpha_{4} .
$$

Denote that

$$
\begin{gathered}
F^{M}=\max _{u \in\left[e^{\alpha_{1}}, e^{\alpha_{4}}\right]} F(u), \quad F^{m}=\min _{u \in\left[e^{\alpha_{1}}, e^{\alpha_{4}}\right]} F(u), \\
g^{m}=\min _{t \in[0, \omega], u \in\left[e^{\alpha_{1}}, e^{\alpha_{4}}\right]} g(t, u) .
\end{gathered}
$$

By $\left(H_{2}\right),\left(H_{3}\right)$, and $\left(H_{9}\right)$, one can easily see that $F^{M}, F^{m}$, and $g^{m}$ are positive constants. Noticing that

$$
e^{\alpha_{1}} \leq e^{x_{1}(t-\tau(t))} \leq e^{\alpha_{4}}
$$

it follows from the first equation of $(27),(30)$, and $\left(H_{3}\right)$ that

$$
\begin{aligned}
F^{m} \leq & \frac{1}{\omega} \int_{0}^{\omega} f\left(t, e^{x_{1}(t-\tau(t))}\right) d t \\
\leq & \frac{e^{x_{2}\left(\eta_{2}\right)}}{\omega} \int_{0}^{\omega} g\left(t, e^{x_{1}(t)}\right) e^{-x_{1}(t)} d t \\
& -\frac{c_{1}}{\omega} \leq \mu e^{x_{2}\left(\eta_{2}\right)}-\frac{c_{1}}{\omega},
\end{aligned}
$$

which implies, by $\left(H_{6}\right)$,

$$
x_{2}\left(\eta_{2}\right) \geq \operatorname{In} \frac{F^{m}+c_{1} / \omega}{\mu} .
$$

Similarly, we also obtain

$$
\begin{aligned}
F^{M} \geq & \frac{1}{\omega} \int_{0}^{\omega} f\left(t, e^{x_{1}(t-\tau(t))}\right) d t \\
\geq & \frac{e^{x_{2}\left(\xi_{2}\right)-x_{1}\left(\eta_{1}\right)}}{\omega} \int_{0}^{\omega} g\left(t, e^{x_{1}(t)}\right) d t \\
& \quad-\frac{c_{1}}{\omega} \geq g^{m} e^{x_{2}\left(\xi_{2}\right)-x_{1}\left(\eta_{1}\right)}-\frac{c_{1}}{\omega},
\end{aligned}
$$

which implies

$$
x_{2}\left(\xi_{2}\right) \leq \ln \frac{F^{M}+c_{1} / \omega}{g^{m}}+x_{1}\left(\eta_{1}\right) \leq \ln \frac{F^{M}+c_{1} / \omega}{g^{m}}+\alpha_{4} .
$$

It follows from the second equation of (29), (40), (42), and Lemma 3 that

$$
\begin{aligned}
x_{2}(t) & \geq x_{2}\left(\eta_{2}\right)-\frac{1}{2}\left(D \omega+\rho_{2}\right) \\
& \geq \ln \frac{F^{m}+c_{1} / \omega}{\mu}-\frac{1}{2}\left(D \omega+\rho_{2}\right):=\alpha_{5}, \\
x_{2}(t) & \leq x_{2}\left(\xi_{2}\right)+\frac{1}{2}\left(R \omega+\rho_{2}\right) \\
& \leq \ln \frac{F^{M}+c_{1} / \omega}{g^{m}}+\alpha_{4}+\frac{1}{2}\left(D \omega+\rho_{2}\right):=\alpha_{6} .
\end{aligned}
$$

In view of (43), we have

$$
\max _{t \in[0, \omega]}\left|x_{2}(t)\right| \leq \max \left\{\left|\alpha_{5}\right|,\left|\alpha_{6}\right|\right\}:=\alpha_{7}
$$

Clearly, $\alpha_{1}, \alpha_{2}, \alpha_{3}, \alpha_{4}$, and $\alpha_{7}$ are independent of $\lambda$.

It is easy to show that algebraic equations

$$
\begin{gathered}
\frac{c_{1}}{\omega}+F\left(e^{x_{1}}\right)-G\left(e^{x_{1}}\right) e^{x_{2}-x_{1}}=0, \\
-\bar{d}+\frac{c_{2}}{\omega}+H\left(e^{x_{1}}\right)=0
\end{gathered}
$$

have two distinct solutions $x_{ \pm}=\left(\ln u_{ \pm}, \ln \left[u_{ \pm}\left(F\left(u_{ \pm}\right)+c_{1} / \omega\right)\right]-\right.$ In $\left.G\left(u_{ \pm}\right)\right)^{T}$. Choose $\alpha_{0}$ such that

$$
\begin{array}{r}
\alpha_{0} \geq \max \left\{\left|\ln \left[u_{-}\left(F\left(u_{-}\right)+\frac{c_{1}}{\omega}\right)\right]-\ln G\left(u_{-}\right)\right|,\right. \\
\left.\left|\ln \left[u_{+}\left(F\left(u_{+}\right)+\frac{c_{1}}{\omega}\right)\right]-\ln G\left(u_{+}\right)\right|\right\} .
\end{array}
$$

We now define

$$
\begin{aligned}
\Omega_{1}=\{ & \left(x_{1}(t), x_{2}(t)\right)^{T} \in X \\
& \left.: x_{1}(t) \in\left(\alpha_{1}, \alpha_{2}\right),\left|x_{2}(t)\right|<\alpha_{7}+\alpha_{0}\right\}, \\
\Omega_{2}=\{ & \left(x_{1}(t), x_{2}(t)\right)^{T} \in X \\
& \left.: x_{1}(t) \in\left(\alpha_{3}, \alpha_{4}\right),\left|x_{2}(t)\right|<\alpha_{7}+\alpha_{0}\right\} .
\end{aligned}
$$


Then both $\Omega_{1}$ and $\Omega_{2}$ are bounded open subsets of $X$. It follows from (36) and (46) that $x_{ \pm} \in \Omega_{i}(i=1,2)$, and $\Omega_{1} \cap$ $\Omega_{2}=\phi$. With the help of (34), (35), (44), and (46), it is easy to see that $\Omega_{i}(i=1,2)$ satisfies condition $(a)$ in Lemma 2 . When $\left(x_{1}(t), x_{2}(t)\right)^{T} \in \partial \Omega_{i} \cap \operatorname{Dom} L=\partial \Omega_{i} \cap R^{2}(i=1,2)$, $\left(x_{1}(t), x_{2}(t)\right)^{T}$ is a constant vector in $R^{2}$. Thus, we have

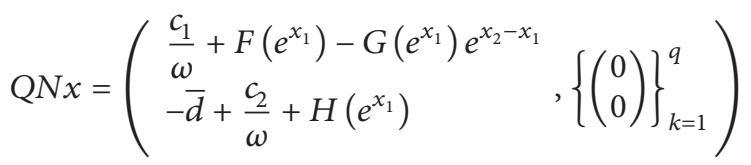

$$
\neq 0
$$

that is, the condition $(b)$ in Lemma 2 holds. In order to verify the condition (c) in Lemma 2 and since the algebraic equations (45) have only one root in $\Omega_{i}(i=1,2)$, let $J$ : $\operatorname{Im} Q \rightarrow \operatorname{Ker} L$ given by $\left(x,(0,0, \ldots, 0)_{2 \times q}\right) \rightarrow x$, where $x \in R^{2}$; in view of the assumptions in Theorem 4 , it is easy to see that

$$
\operatorname{JQN} x=\left(\begin{array}{l}
\frac{c_{1}}{\omega}+F\left(e^{x_{1}}\right)-G\left(e^{x_{1}}\right) e^{x_{2}-x_{1}} \\
-\bar{d}+\frac{c_{2}}{\omega}+H\left(e^{x_{1}}\right)
\end{array}\right),
$$

and a direct computation gives

$$
\begin{gathered}
\operatorname{deg}\left\{J Q N x, \Omega_{i} \cap \operatorname{Ker} L,(0,0)^{T}\right\} \\
=(-1)^{i+1} \neq 0, \quad i=1,2 .
\end{gathered}
$$

By now we have proved that $\Omega_{i}(i=1,2)$ satisfies all the requirements of Lemma 2. Consequently, the system (8) has at least two $\omega$-periodic solutions in $\operatorname{Dom} L \cap \Omega_{i}(i=1,2)$, that is $\left(x_{1}^{*}, x_{2}^{*}\right)$, and $\left(\tilde{x}_{1}, \tilde{x}_{2}\right)$, respectively. Set $x^{*}(t)=e^{x_{1}^{*}(t)}$, $y^{*}(t)=e^{x_{2}^{*}(t)} ; \tilde{x}(t)=e^{\widetilde{x}_{1}(t)}, \tilde{y}(t)=e^{\tilde{x}_{2}(t)}$; then $\left(x^{*}(t), y^{*}(t)\right)^{T}$, $(\tilde{x}(t), \tilde{y}(t))^{T}$ are two positive $\omega$-periodic solutions of the system (4). This completes the proof.

In a weaker condition, we have the following result for the existence of one positive periodic solution.

Theorem 5. In addition to $\left(H_{1}\right)-\left(H_{7}\right)$, suppose further that the following holds:

$$
\left(H_{10}\right) u_{-} e^{1 / 2\left(R \omega+\rho_{1}\right)}<\min \left\{u_{+}, \alpha\right\} .
$$

Then, system (4) has at least one w-periodic solution with strictly positive components.

Proof. The proof is similar to the proof of Theorem 4. Under condition $\left(H_{10}\right)$, (36) is no longer true and $\Omega_{1} \cap \Omega_{2} \neq \phi$. So, we have to make a corresponding change. By $\left(H_{10}\right)$, we can know that

$$
\alpha_{1}<\ln u_{-}<\alpha_{2}<\ln u_{+} \text {. }
$$

Denote that

$$
\begin{gathered}
F^{L}=\max _{u \in\left[e^{\alpha_{1}}, e^{\alpha_{2}}\right]} F(u), \quad F^{l}=\min _{u \in\left[e^{\alpha_{1}}, e^{\alpha_{2}}\right]} F(u), \\
g^{l}=\min _{t \in[0, \omega], u \in\left[e^{\alpha_{1}}, e^{\alpha_{2}}\right]} g(t, u) .
\end{gathered}
$$

By $\left(H_{2}\right),\left(H_{3}\right)$, and $\left(H_{10}\right)$, one can easily see that $F^{L}, F^{l}$, and $g^{l}$ are positive constants. By a similar analysis as that in Theorem 4 , when $x_{1}(t) \in\left(\alpha_{1}, \alpha_{2}\right)$, we have

$$
\begin{aligned}
x_{2}(t) & \geq x_{2}\left(\eta_{2}\right)-\frac{1}{2}\left(D \omega+\rho_{2}\right) \\
& \geq \ln \frac{F^{l}+c_{1} / \omega}{\mu}-\frac{1}{2}\left(D \omega+\rho_{2}\right):=\alpha_{8}, \\
x_{2}(t) & \leq x_{2}\left(\xi_{2}\right)+\frac{1}{2}\left(R \omega+\rho_{2}\right) \\
& \leq \ln \frac{F^{L}+c_{1} / \omega}{g^{l}}+\alpha_{2}+\frac{1}{2}\left(D \omega+\rho_{2}\right):=\alpha_{9} .
\end{aligned}
$$

Thus,

$$
\max _{t \in[0, \omega]}\left|x_{2}(t)\right| \leq \max \left\{\left|\alpha_{8}\right|,\left|\alpha_{9}\right|\right\}:=\alpha_{10}
$$

Clearly, $\alpha_{1}, \alpha_{2}$, and $\alpha_{10}$ are independent of $\lambda$.

It is easy to show that algebraic equations (45) have at least one solution $x_{-}=\left(\ln u_{-}, \ln \left[u_{-}\left(F\left(u_{-}\right)+c_{1} / \omega\right)\right]-\right.$ $\left.\ln G\left(u_{-}\right)\right)^{T}$. We now take $\Omega=\left\{\left(x_{1}(t), x_{2}(t)\right)^{T} \in X: x_{1}(t) \epsilon\right.$ $\left.\left(\alpha_{1}, \alpha_{2}\right),\left|x_{2}(t)\right|<\alpha_{10}+\alpha_{0}\right\}$. It follows from (46) and (51) that $x_{-} \in \Omega$. By a similar analysis as that in Theorem 4 , it is easy to see that $\Omega$ satisfies condition $(a)$ and $(b)$ in Lemma 2 . In order to verify the conditions $(c)$ in Lemma 2 , a direct calculation shows that

$$
\operatorname{deg}\left\{J Q N x, \Omega \cap \operatorname{Ker} L,(0,0)^{T}\right\}=1 \neq 0 .
$$

Hence, $\Omega$ satisfies all the requirements in Lemma 2. Consequently, the system (8) has at least one $\omega$-periodic solution in $\operatorname{Dom} L \cap \bar{\Omega}$, say $\left(x_{1}^{*}, x_{2}^{*}\right)$. Set $x^{*}(t)=e^{x_{1}^{*}(t)}, y^{*}(t)=e^{x_{2}^{*}(t)}$; then $\left(x^{*}(t), y^{*}(t)\right)^{T}$ is one positive $\omega$-periodic solution of the system (4). This completes the proof.

Remark 6. When there is no impulse, that is, $c_{i k} \equiv 0(i=$ $1,2), k=1,2, \ldots, c_{1}=c_{2}=\rho_{1}=\rho_{2} \equiv 0$. The conditions $\left(H_{6}\right)$ and $\left(H_{7}\right)$ are automatically satisfied and $\left(H_{8}\right),\left(H_{9}\right)$, and $\left(H_{10}\right)$ reduce to

$$
\begin{aligned}
& \left(H_{8}^{\prime}\right) u_{-} e^{R \omega}<u_{+} ; \\
& \left(H_{9}^{\prime}\right) u_{+} e^{R \omega / 2}<\alpha ; \\
& \left(H_{10}^{\prime}\right) u_{-} e^{R \omega / 2}<\min \left\{u_{+}, \alpha\right\} .
\end{aligned}
$$

Hence, we have the following corollaries.

Corollary 7. In addition to $\left(H_{1}\right)-\left(H_{5}\right)$, suppose further that $\left(H_{8}^{\prime}\right)$ and $\left(H_{9}^{\prime}\right)$ hold. Then system (2) has at least two positive periodic solutions.

Corollary 8. In addition to $\left(H_{1}\right)-\left(H_{5}\right)$, suppose further that $\left(H_{10}^{\prime}\right)$ holds. Then system (2) has at least one positive periodic solution.

Remark 9. In [4], Ding and Jiang got the following results.

Theorem A. In addition to the conditions $\left(H_{1}\right)-\left(H_{7}\right)$, suppose further that the following conditions hold: 


$$
\begin{aligned}
& \left(A_{1}\right) u_{-} e^{2 R \omega}<u_{+} ; \\
& \left(A_{2}\right) u_{+} e^{R \omega}<\alpha .
\end{aligned}
$$

Then, system (2) has at least two w-periodic solutions with strictly positive components.

Theorem B. In addition to $\left(H_{1}\right)-\left(H_{5}\right)$, suppose further that the following holds:

$$
\left(A_{3}\right) u_{-} e^{R \omega}<\min \left\{u_{+}, \alpha\right\} .
$$

Then, system (2) has at least one w-periodic solution with strictly positive components.

Obviously, the conditions $\left(H_{8}^{\prime}\right),\left(H_{9}^{\prime}\right)$, and $\left(H_{10}^{\prime}\right)$ are weaker than the corresponding $\left(A_{1}\right),\left(A_{2}\right)$, and $\left(A_{3}\right)$, respectively. Hence, our results generalize and improve the corresponding results of [4].

\section{Applications}

In this section, we will list some applications of the previous results.

Application 1. Consider the system (3) which can be obtained by letting

$$
\begin{gathered}
f(t, x)=r(t)-a(t) x, \quad g(t, x)=\frac{b(t) x}{\left(x^{2} / m\right)+x+n}, \\
h(t, x)=\frac{c(t) x}{\left(x^{2} / m\right)+x+n}
\end{gathered}
$$

in system (4). By Theorem 4, we get the following result.

Theorem 10. Suppose that the following conditions hold:

(1) $\bar{d} \omega-c_{2}>0$;

(2) $\bar{c}>\max \left\{(1+2 \sqrt{n / m})\left(\bar{d}-c_{2} / \omega\right) e^{(1 / 2)\left(R \omega+\rho_{1}\right)}-\right.$ $\sqrt{\left(m^{2}\left(\bar{c}-\bar{d}+c_{2} / \omega\right)^{2}-4 m n\left(\bar{d}-c_{2} / \omega\right)^{2}\right)} / m$, $\left.(1+2 \sqrt{n / m})\left(\bar{d}-c_{2} / \omega\right)\right\} ;$

(3) $2\left(\bar{r} \omega+c_{1}\right)\left(\bar{d}-c_{2} / \omega\right)>\bar{a} \omega\left[m\left(\bar{c}-\bar{d}+c_{2} / \omega\right)+\right.$ $\left.\sqrt{m^{2}\left(\bar{c}-\bar{d}+c_{2} / \omega\right)^{2}-4 m n\left(\bar{d}-c_{2} / \omega\right)^{2}}\right] e^{(1 / 2)\left(R \omega+\rho_{1}\right)}$.

Then, system (3) has at least two w-periodic solutions with strictly positive components.

Remark 11. In Theorem 3.2 of [17], Wang et al. proved that system (3) has at least two $\omega$-periodic solutions with strictly positive components under the conditions:

$\left(1^{\prime}\right) \bar{d} \omega-c_{2}>0$

$\left(2^{\prime}\right) \bar{c}>(1+2 \sqrt{n / m})\left(\bar{d}-c_{2} / \omega\right) e^{(1 / 2)\left(R \omega+\rho_{1}\right)}$;

$\left(3^{\prime}\right) 2\left(\bar{r} \omega+c_{1}\right)\left(\bar{d}-c_{2} / \omega\right)>\bar{a} \omega\left[m\left(\bar{c} e^{\left(R \omega+\rho_{1}\right) / 2}-\bar{d}+c_{2} / \omega\right)\right.$ $\left.+\sqrt{m^{2}\left(\bar{c} e^{\left(R \omega+\rho_{1}\right) / 2}-\bar{d}+c_{2} / \omega\right)^{2}-4 m n\left(\bar{d}-c_{2} / \omega\right)^{2}}\right]$ $e^{\left(R \omega+\rho_{1}\right) / 2}$.
Obviously, (2') implies (2). Notice that

$$
\begin{aligned}
m\left(\bar{c} e^{\left(R \omega+\rho_{1}\right) / 2}-\bar{d}+\frac{c_{2}}{\omega}\right) & +\sqrt{m^{2}\left(\bar{c} e^{\left(R \omega+\rho_{1}\right) / 2}-\bar{d}+\frac{c_{2}}{\omega}\right)^{2}-4 m n\left(\bar{d}-\frac{c_{2}}{\omega}\right)^{2}} \\
= & {\left[m\left(\bar{c}-\left(\bar{d}-\frac{c_{2}}{\omega}\right) e^{-\left(R \omega+\rho_{1}\right) / 2}\right)\right.} \\
& +\left(m^{2}\left(\bar{c}-\left(\bar{d}-\frac{c_{2}}{\omega}\right) e^{-\left(R \omega+\rho_{1}\right) / 2}\right)^{2}\right. \\
> & {\left[\begin{array}{l}
m\left(\bar{c}-\bar{d}+\frac{c_{2}}{\omega}\right) \\
\left.\left.(\bar{c})^{2} e^{-\left(R \omega+\rho_{1}\right)}\right)^{1 / 2}\right] e^{\left(R \omega+\rho_{1}\right) / 2}
\end{array}\right.} \\
& \left.+\sqrt{m^{2}\left(\bar{c}-\bar{d}+\frac{c_{2}}{\omega}\right)^{2}-4 m n\left(\bar{d}-\frac{c_{2}}{\omega}\right)^{2}}\right] e^{(1 / 2)\left(R \omega+\rho_{1}\right)} .
\end{aligned}
$$

Thus, $\left(3^{\prime}\right)$ also implies (3). Hence, Theorem 10 improves Theorem 3.2 in [17].

Application 2. Consider the following system:

$$
\begin{gathered}
x^{\prime}(t)=x(t)\left[\frac{r(t)-a(t) x(t-\tau(t))}{n+x(t-\tau(t))}\right. \\
\left.-\frac{b(t) y\left(t-\sigma_{1}(t)\right)}{x^{2}(t)+m^{2}}\right], \\
y^{\prime}(t)=y(t)\left[\frac{c(t) x\left(t-\sigma_{2}(t)\right)}{x^{2}\left(t-\sigma_{2}(t)\right)+m^{2}}-d(t)\right], \quad t \neq t_{k}, \\
\Delta x(t)=x\left(t^{+}\right)-x(t)=c_{1 k} x(t), \\
\Delta y(t)=y\left(t^{+}\right)-y(t)=c_{2 k} y(t), \quad t=t_{k},
\end{gathered}
$$

which isz a special form of system (4) by replacing

$$
\begin{gathered}
f(t, x)=\frac{r(t)-a(t) x}{n+x}, \quad g(t, x)=\frac{b(t) x}{m^{2}+x^{2}}, \\
h(t, x)=\frac{c(t) x}{m^{2}+x^{2}} .
\end{gathered}
$$

The prey population follows the Smith [24] model.

By Theorem 4, we have the following result.

Theorem 12. Suppose that the following conditions hold:

(1) $\bar{d} \omega-c_{2}>0$;

(2) $\bar{c}>\max \left\{2 m\left(\bar{d}-c_{2} / \omega\right) e^{(1 / 2)\left(R \omega+\rho_{1}\right)} \quad-\right.$ $\left.\sqrt{\bar{c}^{2}-4 m^{2}\left(\bar{d}-c_{2} / \omega\right)^{2}}, 2 m\left(\bar{d}-c_{2} / \omega\right)\right\} ;$ 


$$
\text { (3) } \begin{aligned}
2\left(\bar{r} \omega+c_{1}\right)\left(\bar{d}-c_{2} / \omega\right)>\bar{a} \omega\left(\bar{c}+\sqrt{\bar{c}^{2}-4 m^{2}\left(\bar{d}-c_{2} / \omega\right)^{2}}\right) \\
e^{(1 / 2)\left(R \omega+\rho_{1}\right)}
\end{aligned}
$$

Then, system (59) has at least two $\omega$-periodic solutions with strictly positive components.

Application 3. Consider the following system:

$$
\begin{gathered}
x^{\prime}(t)=x(t)\left[r(t)-a(t) x(t-\tau(t))-e(t) x^{2}(t-\tau(t))\right] \\
-\frac{b(t) x(t) y\left(t-\sigma_{1}(t)\right)}{x^{2}(t)+m^{2}}, \\
y^{\prime}(t)=y(t)\left[\frac{c(t) x\left(t-\sigma_{2}(t)\right)}{m^{2}+x^{2}\left(t-\sigma_{2}(t)\right)}-d(t)\right], \quad t \neq t_{k}, \\
\Delta x(t)=x\left(t^{+}\right)-x(t)=c_{1 k} x(t), \\
\Delta y(t)=y\left(t^{+}\right)-y(t)=c_{2 k} y(t), \quad t=t_{k},
\end{gathered}
$$

which is a special case of (4) by letting

$$
\begin{gathered}
f(t, x)=r(t)-a(t) x-e(t) x^{2}, \\
g(t, x)=\frac{b(t) x}{m^{2}+x^{2}}, \quad h(t, x)=\frac{c(t) x}{m^{2}+x^{2}},
\end{gathered}
$$

where functions $r(t), a(t), b(t), c(t), d(t), \tau(t), \sigma_{1}(t), \sigma_{2}(t)$ and constant $m$ are defined as above, $e(t)$ is a positive continuous $\omega$-periodic function, and the prey population follows the Allee effect [25] model. By Theorem 4, we have the following result.

Theorem 13. Suppose that the following conditions hold:

(1) $\bar{d} \omega-c_{2}>0$;

(2) $\bar{c}$

$$
\begin{aligned}
& \bar{c} \quad>\quad \max \left\{2 m\left(\bar{d}-c_{2} / \omega\right) e^{(1 / 2)\left(Z \omega+\rho_{1}\right)}-\right. \\
& \left.\sqrt{\bar{c}^{2}-4 m^{2}\left(\bar{d}-c_{2} / \omega\right)^{2}}, 2 m\left(\bar{d}-c_{2} / \omega\right)\right\} ;
\end{aligned}
$$

(3) $2 \alpha\left(\bar{d}-c_{2} / \omega\right)>\left(\bar{c}+\sqrt{\bar{c}^{2}-4 m^{2}\left(\bar{d}-c_{2} / \omega\right)^{2}}\right) e^{(1 / 2)\left(Z \omega+\rho_{1}\right)}$,

Then, system (61) has at least two $\omega$-periodic solutions with strictly positive components, where

$$
\alpha=\frac{\bar{a}+\sqrt{\bar{a}^{2}+4 \overline{e r}}}{2 \bar{e}}, \quad z(t)=\frac{a^{2}(t)+4 e(t) r(t)}{4 e(t)} .
$$

\section{Conflict of Interests}

The authors declare that there is no conflict of interests regarding the publication of this paper.

\section{Acknowledgments}

The research is supported by NSF of China (nos. 11161015, 11371367, and 11361012), PSF of China (nos. 2012M512162 and 2013T60934), NSF of Hunan Province (nos. 11JJ900, 12JJ9001, and 13JJ4098), the Education Foundation of Hunan province (nos. 12C0541, 12C0541, and 13C084), and the Construct Program of the Key Discipline in Hunan province.

\section{References}

[1] R. E. Gaines and J. L. Mawhin, Coincidence Degree, and Nonlinear Differential Equations, Springer, Berlin, Germany, 1977.

[2] Y. M. Chen, "Multiple periodic solutions of delayed predatorprey systems with type $I V$ functional responses," Nonlinear Analysis: Real World Applications, vol. 5, no. 1, pp. 45-53, 2004.

[3] D. M. Xiao and S. G. Ruan, "Multiple bifurcations in a delayed predator-prey system with nonmonotonic functional response," Journal of Differential Equations, vol. 176, no. 2, pp. 494-510, 2001.

[4] X. Q. Ding and J. F. Jiang, "Multiple periodic solutions in generalized Gause-type predator-prey systems with non-monotonic numerical responses," Nonlinear Analysis: Real World Applications, vol. 10, no. 5, pp. 2819-2827, 2009.

[5] X. L. Hu, G. R. Liu, and J. R. Yan, "Existence of multiple positive periodic solutions of delayed predator-prey models with functional responses," Computers \& Mathematics with Applications, vol. 52, no. 10-11, pp. 1453-1462, 2006.

[6] Y. H. Xia, J. D. Cao, and S. S. Cheng, "Multiple periodic solutions of a delayed stage-structured predator-prey model with nonmonotone functional responses," Applied Mathematical Modelling, vol. 31, no. 9, pp. 1947-1959, 2007.

[7] Z. G. Luo and J. H. Shen, "New Razumikhin type theorems for impulsive functional differential equations," Applied Mathematics and Computation, vol. 125, no. 2-3, pp. 375-386, 2002.

[8] G. Ballinger and X. Z. Liu, "Existence, uniqueness and boundedness results for impulsive delay differential equations," Applicable Analysis, vol. 74, no. 1-2, pp. 71-93, 2000.

[9] B. Liu, Z. D. Teng, and W. B. Liu, "Dynamic behaviors of the periodic Lotka-Volterra competing system with impulsive perturbations," Chaos, Solitons and Fractals, vol. 31, no. 2, pp. 356-370, 2007.

[10] D. Bănov and P. Simeonov, Impulsive Differential Equations: Periodic Solutions and Applications, vol. 66, Longman Sci. Tech., Harlow, UK, 1993.

[11] V. Lakshmikantham, D. Bainov, and P. Simeonov, Theory of Impulsive Dfferential Equations, vol. 6, World Scientific Publishing, Teaneck, NJ, USA, 1989.

[12] Z. G. Luo, B. X. Dai, and Q. Wang, "Existence of positive periodic solutions for a nonautonomous neutral delay $n$-species competitive model with impulses," Nonlinear Analysis: Real World Applications, vol. 11, no. 5, pp. 3955-3967, 2010.

[13] X. D. Li and X. L. Fu, "On the global exponential stability of impulsive functional differential equations with infinite delays or finite delays," Communications in Nonlinear Science and Numerical Simulation, vol. 19, no. 3, pp. 442-447, 2013.

[14] Z. G. Luo and L. P. Luo, "Existence and stability of positive periodic solutions for a neutral multispecies Logarithmic population model with feedback control and impulse," Abstract and Applied Analysis, vol. 2013, Article ID 741043, 11 pages, 2013.

[15] Z. G. Luo and L. P. Luo, "Global positive periodic solutions of generalized $n$-species competition systems with multiple delays and impulses," Abstract and Applied Analysis, vol. 2013, Article ID 980974, 13 pages, 2013.

[16] M. Liu and K. Wang, "Asymptotic behavior of a stochastic nonautonomous Lotka-Volterra competitive system with impulsive perturbations," Mathematical and Computer Modelling, vol. 57, no. 3-4, pp. 909-925, 2013. 
[17] Q. Wang, B. X. Dai, and Y. M. Chen, "Multiple periodic solutions of an impulsive predator-prey model with Hollingtype IV response," Mathematical and Computer Modelling, vol. 49, no. 9-10, pp. 1829-1836, 2009.

[18] B. X. Dai, H. Su, and D. W. Hu, "Periodic solution of a delayed ratio-dependent predator-prey model with monotonic functional response and impulse," Nonlinear Analysis: Theory, Methods \& Applications, vol. 70, no. 1, pp. 126-134, 2009.

[19] S. M. Moghadas and B. D. Corbett, "Limit cycles in a generalized Gause-type predator-prey model," Chaos, Solitons and Fractals, vol. 37, no. 5, pp. 1343-1355, 2008.

[20] X. Q. Ding and J. F. Jiang, "Positive periodic solutions in delayed Gause-type predator-prey systems," Journal of Mathematical Analysis and Applications, vol. 339, no. 2, pp. 1220-1230, 2008.

[21] Y. Kuang and E. Beretta, "Global qualitative analysis of a ratiodependent predator-prey system," Journal of Mathematical Biology, vol. 36, no. 4, pp. 389-406, 1998.

[22] J. F. Andrews, "A mathematical model for the continuous cul ture of microorgnaisms utilizing inhibitory substrates," Biotechnology and Bioengineering, vol. 10, pp. 707-723, 1986.

[23] W. Sokol and J. A. Howell, "Kinetics of phenol oxidation by washed cells," Biotechnology and Bioengineering, vol. 23, pp. 2039-2049, 1980.

[24] F. E. Smith, "Population dynamics in daphnia magna and a new model for population growth," Ecology, vol. 44, pp. 651-663, 1963.

[25] K. Gopalsamy and G. Ladas, "On the oscillation and asymptotic behavior of $\dot{N}(t)=N(t)\left[a+b N(t-\tau)-c N^{2}(t-\tau)\right]$," Quarterly of Applied Mathematics, vol. 3, pp. 433-440, 1990. 


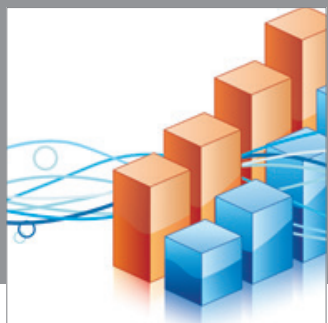

Advances in

Operations Research

mansans

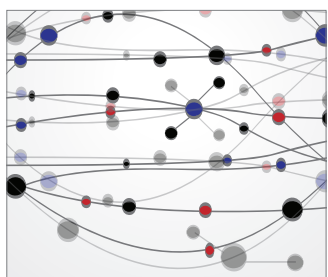

The Scientific World Journal
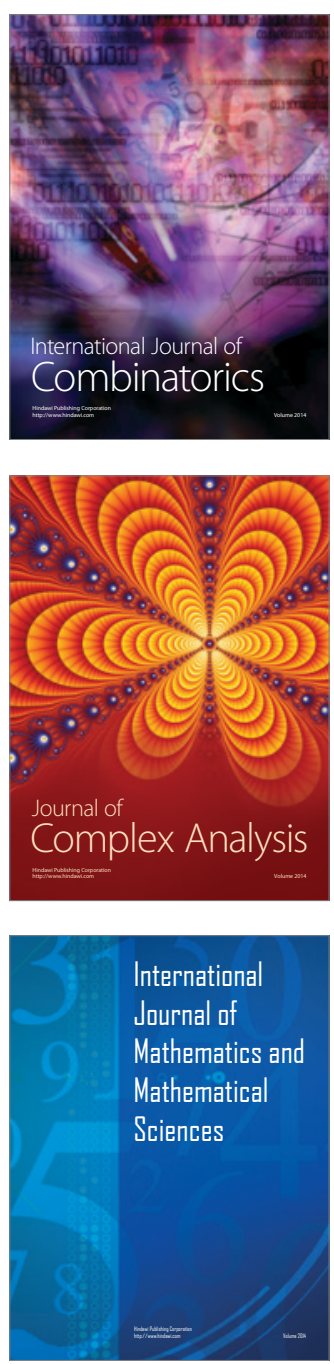
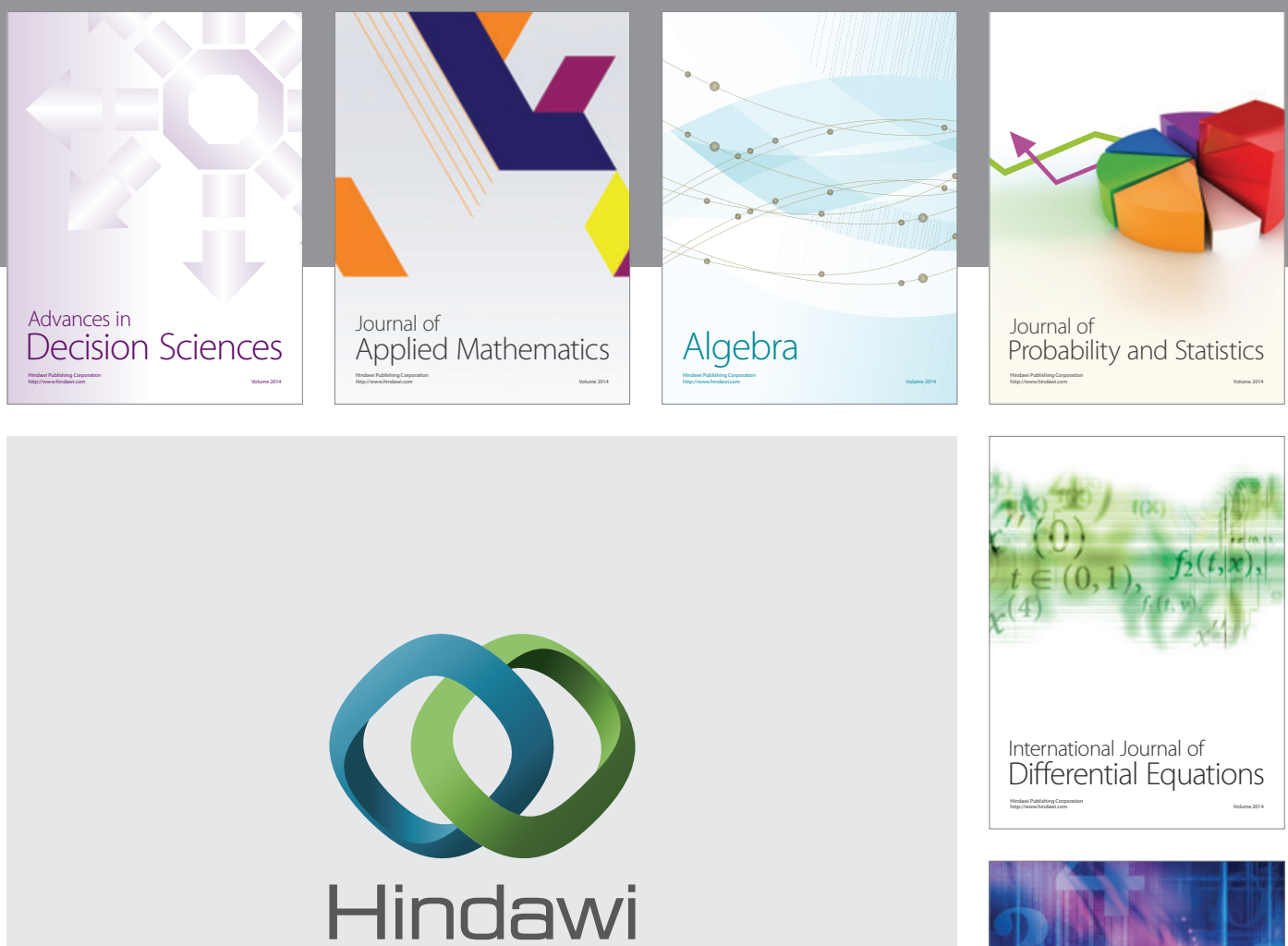

Submit your manuscripts at http://www.hindawi.com
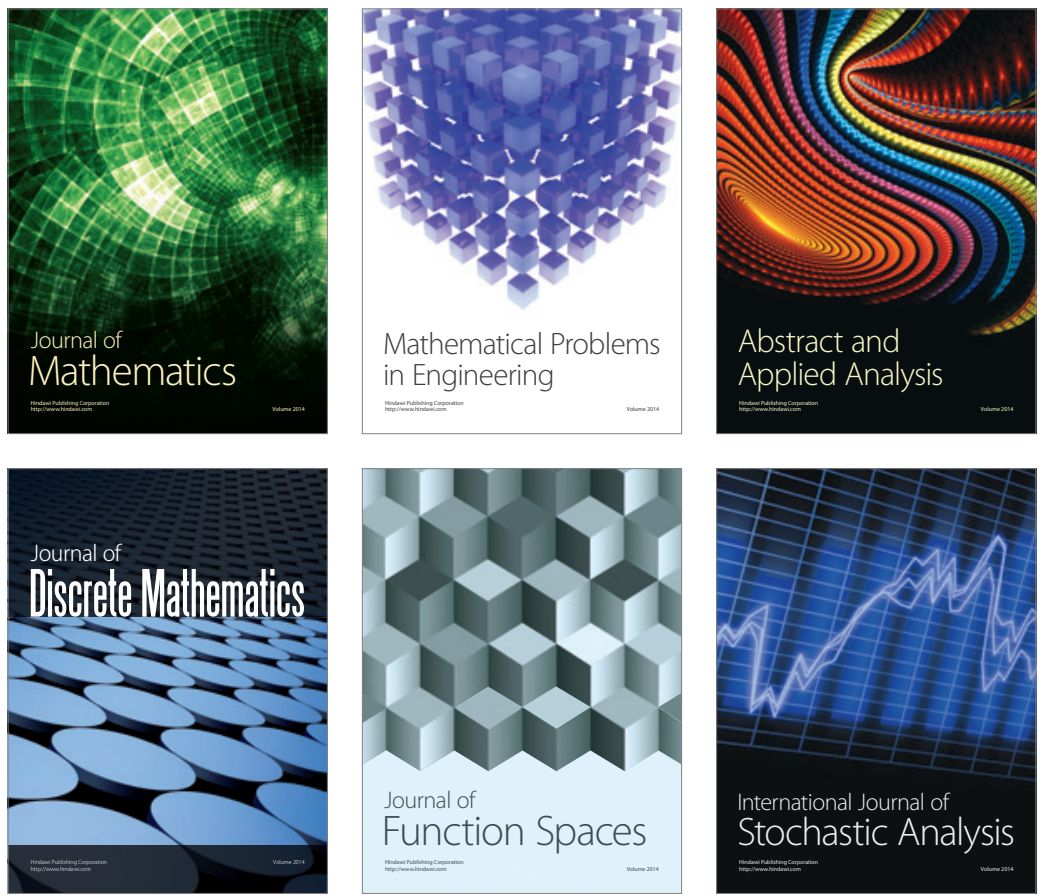

Journal of

Function Spaces

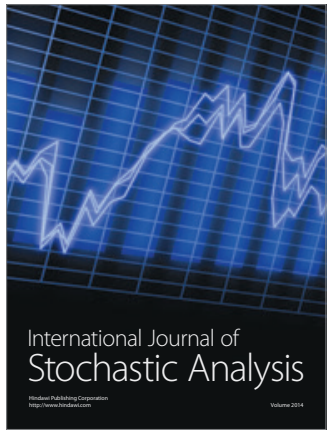

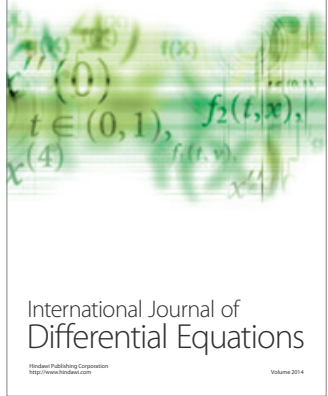
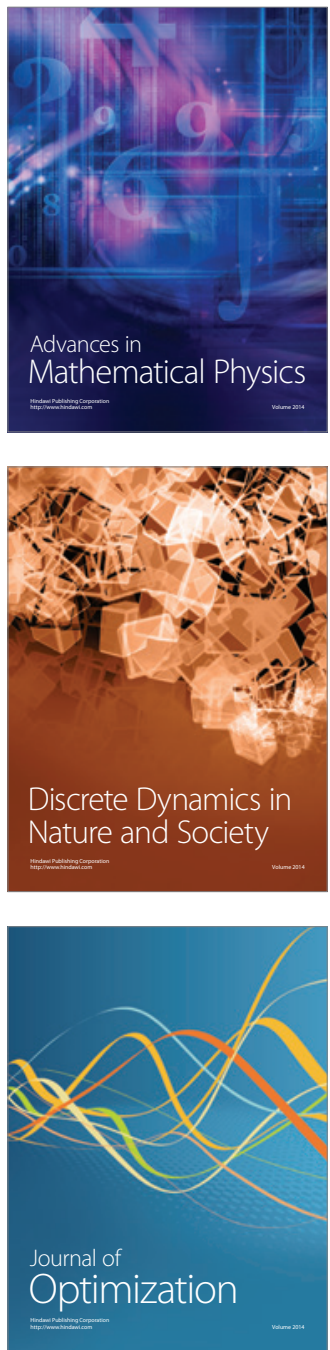